\title{
Atomistic Simulation to Study the Fracture Behaviour in Cu-Zr Bulk Metallic Glass
}

\author{
Rahul Chug $^{\# 1}$, Sachin Gupta*2 \\ \# Department of Mechanical \& Automation Engineering., \\ Maharaja Agrasen Institute of Technology, Rohini sector-22, New Delhi-110086, India \\ ${ }^{1}$ rahulchughmech@gmail.com \\ * Department of Mechanical \& Automation Engineering., \\ Maharaja Agrasen Institute of Technology, Rohini sector-22, New Delhi-110086, India \\ 2 coolprofessor83@gmail.com
}

\begin{abstract}
In spite of its critical importance for the use of Bulk Metallic Glasses (BMGs) as structural materials, it has been observed in literature that fracture toughness of BMGs is highly variable and also the fracture toughness of BMGs is relatively poorly understood. In the present study, fracture behavior of monolithic Cu-Zr BMG has been studied using Molecular Dynamics (MD) simulation with Embedded Atom Method (EAM) potentials. Four different specimens with increasing sizes were used to investigate the influences of material length scale on stress-strain and fracture behavior of Cu-Zr BMG. Additionally, the effect of temperature, role of addition of $\mathrm{Al}$ and the loading strain rate on the stress-strain behaviour were also examined. In the simulation of each specimen, the averaged stress-strain curve was observed. The simulation results show that although the initial slope of the averaged stress-strain curve is independent of the specimen size, temperature and strain rate, they can strongly influence the peak stress and thereby the crack growth.
\end{abstract}

Keywords-Fracture Mechanics, Bulk Metallic Glass, MD Simulation

\section{INTRODUCTION}

This Recent remarkable progress in manufacturing technology enables us to produce atomic-scale materials, the structure of which can precisely be controlled at an atom-by-atom level, e.g. CNT, gold nanowire etc. To ensure reliability, nano-components should be carefully designed on the basis of mechanical conditions. However, the fracture mechanism in nanocrystalline materials is not clearly understood [1]. To make known the mechanism of fracture at the nano-scale requires discerning the evolution process of the crack tip under the application of increased stress intensity, which is strongly affected by the local atomic structure, lattice orientation, inter-atomic potentials, and generation of defects [2].

Over the last few decades, significant progress in computing power enables us to analyze the mechanical behavior of materials at the atomic level by using numerical calculations such as MD simulations. Computer simulation of fracture in single crystals has provided new insight into the stability of crack propagation, phenomena of lattice trapping, and the origins of brittle and ductile behavior. MD simulations predict the motion of atoms in an atomistic system through Newton's equations of motions. These equations, combined with the initial and boundary conditions, can be solved using numerical integration method to obtain the trajectories of the atoms such as the position and the velocity as a function of time [2]. Often the inter-atomic potential is a classical potential, no quantum mechanical description of the material is attempted, but the functional form of the potential may be derived from quantum mechanical arguments. MD is able to treat systems with millions of atoms, and permits the average calculations of transport or mechanical quantities and also the modeling of complex phenomena like shear band localization, fracture appearance etc. The quality of results depends on the quality of the interatomic potential; simple potentials provide less accurate description of the interatomic interactions while allowing very large simulations whereas more complicated potentials may give a better description of the interaction, but limit the simulation size. Many-body potentials such as the EAM and the Effective Medium Theory have been shown to give a good description of the late transition metals crystallizing in closed-packed structures, and their alloys, while still allowing simulations with millions of atoms [3].

A lot of work has been done on MD simulation in fracture mechanics, from early 90's to till date. Majority of the work have been focused on fracture behavior of nanocrystalline metals and alloys. These studies include the fast brittle fracture in a material [4-7], instability of dynamic fracture in three-dimensional fracture[8-9], brittle-to-ductile transition in crack growth [10-11], stress-induced phase transformations and grain nucleation at crack tip [12],influences of grain boundary [13-14], and large-scale MD simulation of three-dimensional fracture [15], crack propagation in fcc metallic plates [16], ductile crack growth in an aluminum single crystal [17], size effect study of magnesium single crystal [2,18] etc. Thus there has been considerable recent research on various nanocrystalline metals like $\mathrm{Cu}, \mathrm{Al}, \mathrm{Mg}$ etc. and some alloys. However, only a few studies, research efforts have been carried out till now to understand the origins of toughness in BMGs [3, 19-24]. 
BMGs are a new class of structural materials that exhibit extraordinary strengths while their elastic moduli are only marginally lower than their crystalline counterparts. The mechanical properties of BMGs are remarkably different from the ones of ordinary metallic alloys due to the atomic level disorder in the glassy state. Unlike crystalline materials plastic deformation in metallic glasses cannot be caused by lattice defects but takes place through atomic scale deformation events and may furthermore involve localization through formation of shear bands. As a result, there has been considerable recent research focused on finding alloy compositions that have excellent glass forming ability as well as mechanical properties. Computational investigations of the deformation response of BMG provide unique insights that complement experiments such as uniaxial tension/ compression tests and instrumented indentation [19].For understanding the origin of their mechanical properties it is important to get the basic understanding of fundamental theoretical problems through atomistic simulations. $\mathrm{Cu}-\mathrm{Zr}$ was recently discovered to be a binary BMG. Since binary alloys are easier to model than alloys with more elements, this makes $\mathrm{Cu}-\mathrm{Zr}$ an attractive BMG to study theoretically [3].

In the present study, effects of specimen size, temperature, role of addition of $\mathrm{Al}$ and loading strain rate on the strength behavior of monolithic Cu-Zr BMG have been examined using MD simulation with EAM potentials. Four different specimens with increasing sizes were used to investigate the influences of material length scale on stress-strain and crack growth behavior of Cu-Zr BMG. The effects of temperature and loading strain rate were also additionally tested. All the specimens were subjected to uniaxial tensile loading. The averaged stress-strain curve was examined for each specimen. The simulation results show that although the initial slope of the averaged stress-strain curve is independent of the specimen size, temperature and strain rate, they can strongly influence the peak stress and consequently the crack growth.

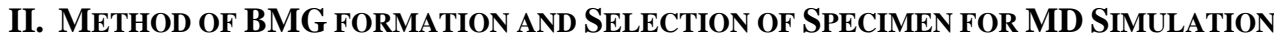

For the purpose of simulation, initially $\mathrm{Cu}_{50} \mathrm{Zr}_{50}$ BMG structures were generated computationally by means of the melt quench procedure using LAMMPS software [25]. First the atoms were randomly arranged in a supercell, where periodic boundary condition was applied in all 3 dimensions, and then heated it up to above the melting temperature. After staying at $2300 \mathrm{~K}$ for $40 \mathrm{ps}$, it was cooled down to $300 \mathrm{~K}$ by rapid quench of cooling rate $10^{14} \mathrm{~K} / \mathrm{s}$. Any phase separation in the cooling process was not observed.

To identify the influence of size effects on the strength behaviour and crack growth, four different specimens of increasing size were studied as shown in Fig. 1. The sizes of the four specimens were generated by increasing the width and the height of the specimen but maintaining the thickness as a constant. An edge crack was introduced in each specimen by maintaining the ratio of the initial crack length to the width of the specimen i.e. $a_{o} / w=0.1$. The resulting number of atoms in each specimen was varied from 5,488 to 87,024 . Table 1 presents the geometrical dimensions and the resulting number of atoms in the four specimens of increasing size. Let, $\mathrm{x}, \mathrm{y}$ and $\mathrm{z}$ coordinate axes represent the [ $\left[\begin{array}{lll}1 & 0 & 0\end{array}\right],\left[\begin{array}{lll}0 & 1 & 0\end{array}\right]$ and $\left[\begin{array}{lll}0 & 0 & 1\end{array}\right]$ crystallographic directions, respectively.

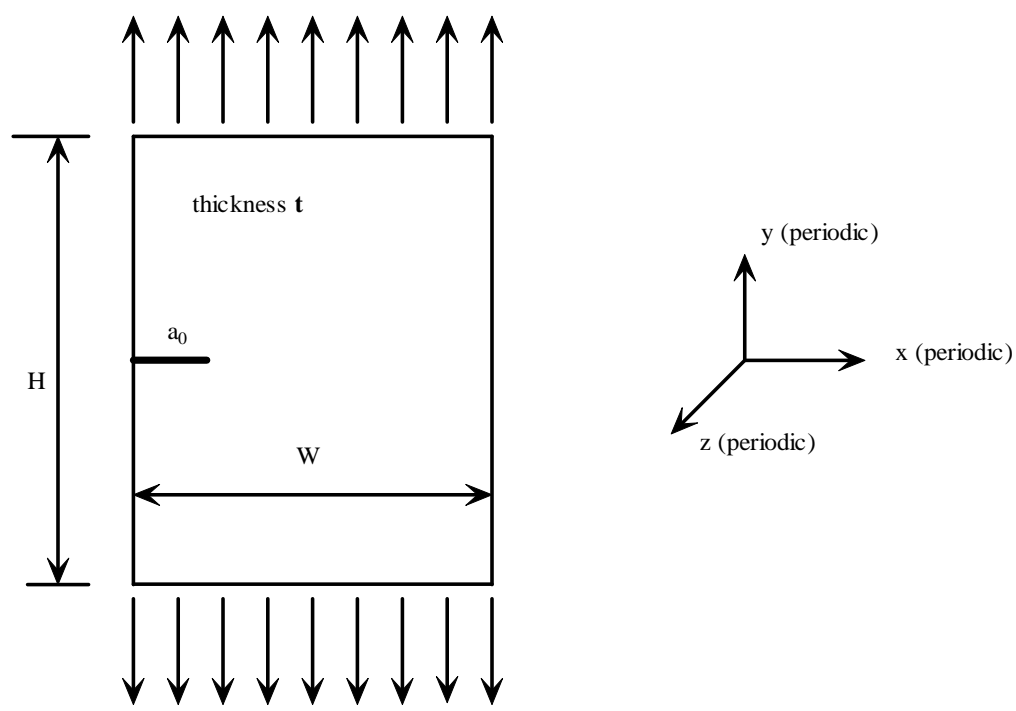

Fig.1. Model geometry of Cu-Zr BMG single crystal containing an edge crack 
TABLE I . Geometrical Dimensions and the Resulting Number of Atoms of the Specimens of Increasing Size

\begin{tabular}{ccccc}
\hline Specimen No. & W $(\dot{\boldsymbol{A}})$ & H $(\dot{\boldsymbol{A}})$ & $\mathbf{T}(\dot{\boldsymbol{A}})$ & Number of atoms \\
\hline $\mathbf{1}$ & 25 & 50 & 50 & 5,488 \\
$\mathbf{2}$ & 50 & 100 & 50 & 21,952 \\
$\mathbf{3}$ & 75 & 150 & 50 & 49,392 \\
$\mathbf{4}$ & 100 & 200 & 50 & 87,024 \\
\hline
\end{tabular}

\section{III.SiMULATION PROCEDURE}

All This section addresses some of the basic MD simulation issues associated with the present computational model. Fig. 1 shows a MD simulation model of single crystal Cu-Zr BMG. All the specimens have free surfaces in the thickness direction (i.e. $\mathrm{z}$ direction). The periodic boundary conditions were assigned in all the 3 directions. In this study, the interactions between the atoms are described by the EAM potential developed by Howard Sheng et al. [25]. Among many proposed inter-atomic potentials EAM potential uses the local electron density as a key variable to describe the inter-atomic bonding in metallic system. It incorporates the influences of local environment, such as surface and defects, on the strength of individual bonds so that it is suitable to be applied to the simulations of fracture and damage. In all the simulations, the MD models are first equilibrated for $4 \mathrm{ps}$ in which the normal stress components are kept at zero and the system temperature is maintained at 300 $\mathrm{K}$ before applying uniaxial tension loading. The uniaxial strain loading in the y direction is applied by changing the corresponding simulation box length at a constant strain rate $\dot{\varepsilon}=v_{y} / L_{y}^{0}$, where $v_{y}$ and $L_{y}^{0}$ are the rate of change of the box length and the initial box length in the y-direction after the equilibrium, respectively. Except in the investigation of strain rate effects, the uniaxial tension loading was applied at a constant strain rate of 0.5 $\mathrm{x} 10^{12} \mathrm{~s}^{-1}$. Similarly, all simulations were performed at a constant temperature of $300 \mathrm{~K}$ except in the studies of the effects of temperature.The thermal expansion of the model was isotropic. Constant NPT integration was performed upon the group of atoms to update the positions and velocities at each time step. All MD simulations were conducted with a time step of $0.001 \mathrm{ps}$.

\section{IV.RESULTS AND DISCUSSION}

In this work, classical MD simulations were performed for BMG subjected to uniaxial tensile strain loading with the parallel MD code of LAMMPS.The reliability of the present numerical study is initially tested by energy conservation graph. Thereafter this section presents the simulation results for the effects of the specimen size, temperature, and addition of $\mathrm{Al}$ and strain rate on the strength and fracture behavior in $\mathrm{Cu}-\mathrm{Zr} \mathrm{BMG}$. The details of the simulation process have been discussed in section 3 .

\section{A. Validation of the Simulation}

As Large Atomic Molecular Massively Parallel Simulator (LAMMPS) was used for the atomistic simulation, the first issue which comes up is its validation. The validation of the simulation technique can be checked by few of the results obtained during the start of the simulation.Energy conservation is one of the most important considerations. The energy conservation graph has been plotted in Fig 2. The graph shows that though there is an initial drift in the total energy, after few time steps, the energy conservation is quite satisfactory.Energy conservation has shown that the time step chosen for the simulation is appropriate. As a consequence, the result obtained in Fig. 2 has been considered sufficient to continue further investigations. 


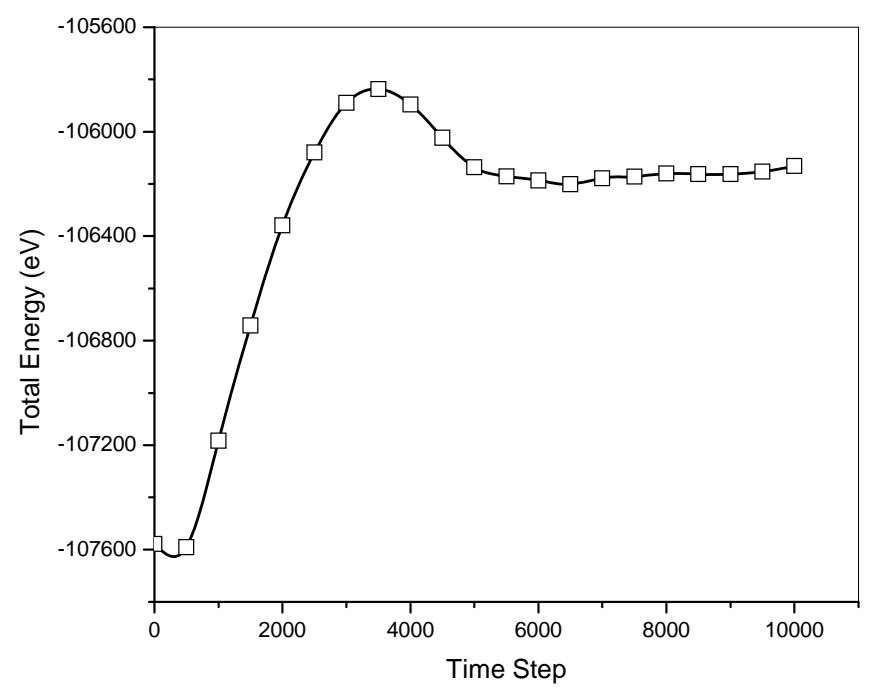

Fig.2. Variation of total energy vs. time step

\section{B. Deformation and Fracture Features}

In general, in the literature it has been observed that the material length scale has significant influences on strength, toughness, ductility and the stress-strain response of materials. Many experimental and simulation studies have shown the strength hardening with decreasing specimen size. A concept of self-similar hardening i.e. smaller is stronger is generally accepted for nanostructures. The dislocation multiplication and volumetric source exhaustion are frequently used to explain this size effect. However, on the other hand, the "smaller is softer" effect has been observed recently by Marian and Knap [26]. Here, in the present study, the average stress-strain curves of four different specimen sizes, as listed in Table 1, obtained from MD simulation are plotted in Fig. 3.

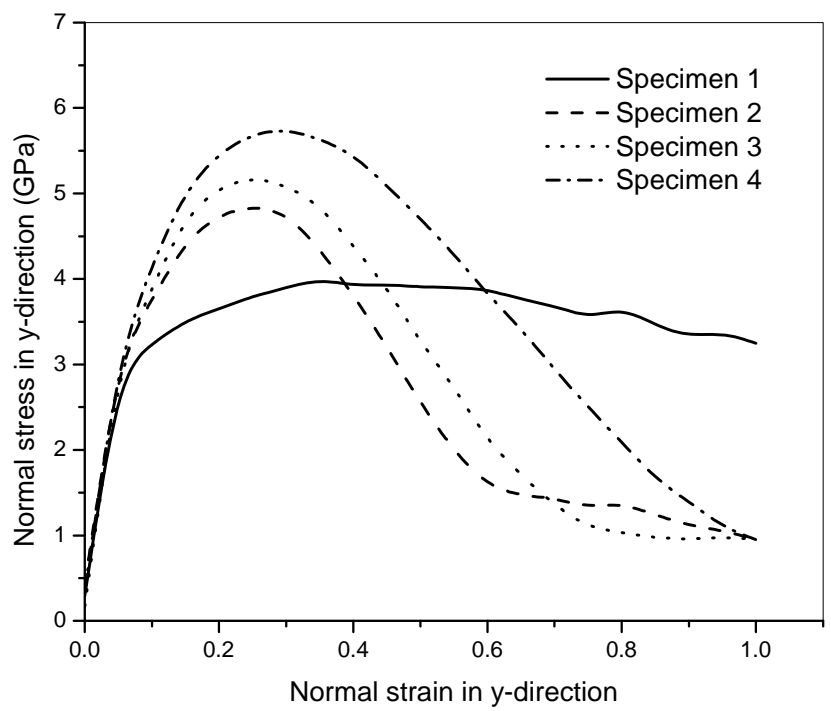

Fig.3.Influence of specimen size on averaged stress-strain response of monolithic Cu-Zr BMG specimen containing an initial edge crack

The initial yield strength is increased with increasing specimen size. The peak stress point for the smallest specimen size is about $4 \mathrm{GPa}$ while it increased to about 5.8 GPa at the largest specimen size. The initial slope of the average stress-strain curve is independent of the specimen size. It has also been found that for the present BMG with the same compositions, the smallest size specimen is much softer than those with larger dimensions; maximum plastic deformation was induced for the smallest dimension. However, for the other three specimen sizes, toughness is increasing with increasing size. The reason for smallest dimension exhibits better plasticity may be due to more free volume content. Also it may be due to the presence of prominent serrations and multiple shear bands. Similar kinds of results were observed in some earlier studies [ 22, 27-29]. So, it appears that some critical length calculation may be required in order to draw any conclusion in the case of Cu-Zr BMG. 
In Fig.3 it has been observed that the material length scale has considerable influences on strength, toughness, ductility and the stress-strain response of materials. In order to illustrate the role of thickness on the above properties a separate study has been carried out. Fig. 4 shows the simulated ultimate strength of $\mathrm{Cu}-\mathrm{Zr} \mathrm{BMG}$ as a function of thickness for the specimen 2. Here, the peak stress point is increasing with increase in thickness of the specimen. Similar kinds of results were also obtained in [29].

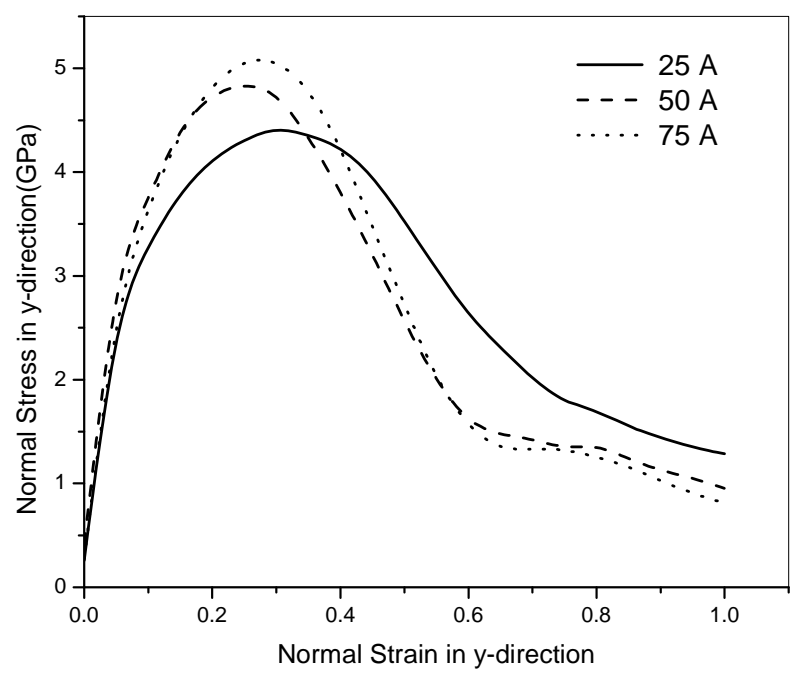

Fig.4. Stress-Strain plot for different thickness for specimen 2 of monolithic Cu-Zr BMG

In the above figures, the simulations were carried out at a constant temperature of $300 \mathrm{~K}$. It is well known that temperature has significant influences on the material properties. To study the effects of temperatures, the specimen 2 in Table 1 i.e. of dimension $\mathrm{W}(50 \dot{A}), \mathrm{H}(100 \dot{A})$ and t $(50 \dot{A})$ has been selected for atomistic model at different temperatures. Fig. 5 shows the averaged stress-strain curves at various temperatures. It is seen that the initial slopes of the uniaxial stress strain curves are independent of temperature. The peak stress point increases with increasing temperature, however the toughness decreases with increase in temperature. Generally, it is believed that the temperature softening results from the weaker bonds between the atoms caused by the increasing temperature. The phenomenon observed in $\mathrm{Cu}-\mathrm{Zr}$ BMG implies that the critical stress of twin nucleation increases with increasing temperature. At higher temperature, the stress concentration at the crack tip was significantly increased by the reorganization of atoms due to the high mobility of atoms at high temperature. It is very clear that increase in temperature causes more blunting and crack arrest. This is in opposition to the conjecture that thermal disturbance would weaken the interatomic bond forces. It is interesting to note that Warner and Curtin [30] revealed similar simulation results where the twining re-emerged in Fe single crystal at high temperature.

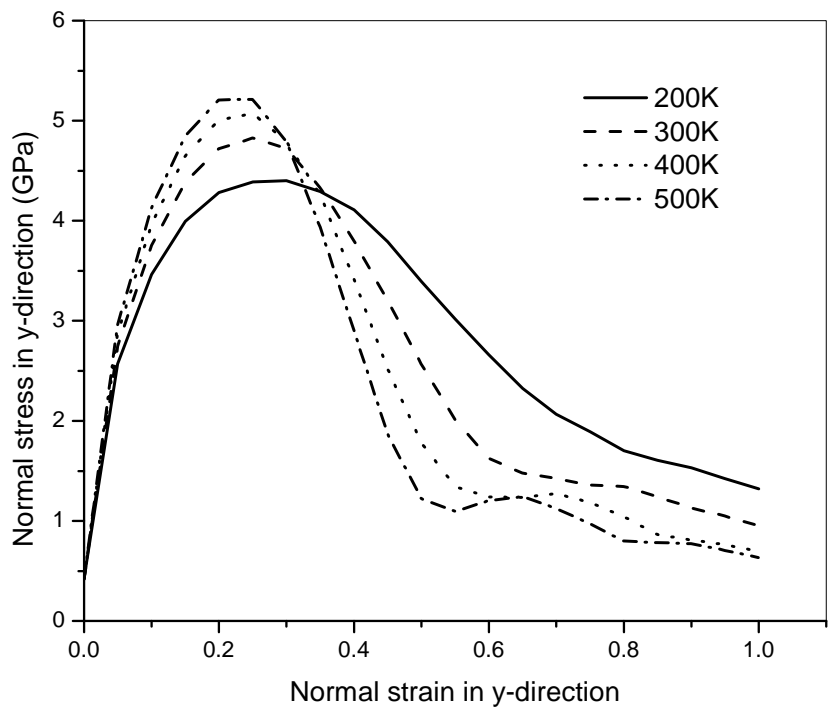

Fig.5. Influence of temperature on averaged stress-strain response of monolithic Cu-Zr BMG specimen containing an initial edge crack 


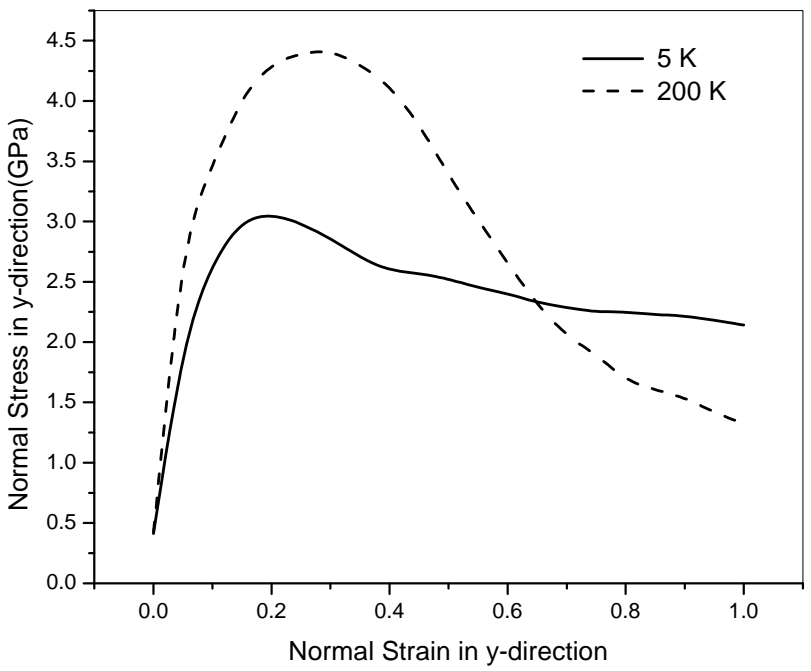

Fig.6. Influence of very low temperature on averaged stress-strain response of monolithic Cu-Zr BMG specimen containing an initial edge crack

In many practical applications study of material behavior at very low temperature is very important such as in cryogenics. Figure 6 describes the behavior of the stress-strain plot of specimen 2 at two different temperatures; one at $5 \mathrm{~K}$ and the other is at $200 \mathrm{~K}$. The low temperature study reveals that though the peak stress point value is low as compared to that of the high temperature the plastic deformation obtained is very high. The explanation of this phenomenon is as yet obscure. This interesting finding may attract the future researchers to explore more on the interesting features of BMG.

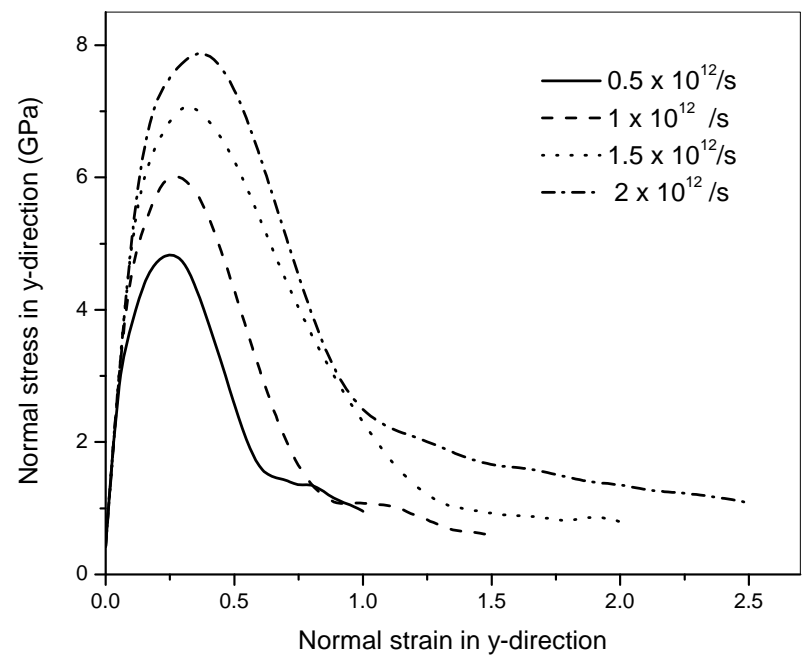

Fig.7.Stress-Strain plot at different loading strain rates

In the literature, generally, it is observed that typical strain rates used in MD simulations are in the range of $10^{7}$ to $10^{11} \mathrm{~s}^{-1}$, which makes the MD method particularly well suited to model material behavior under extremely-high strain rate loading. In order to observe the influence of strain rate on the peak stress value and the plastic deformation the stress-strain plots for four different loading strain rates have been plotted for the same specimen 2, Table 1 in Fig. 7. The figure illustrates that a higher strain rate yields a much higher strength, whereas the modulus is much less sensitive to the loading rate. This strain rate effect can be explained by the disorder of the crystal lattice due to external loading [31,32]. A higher strain rate could lead to a greater degree of lattice disorder and the crystal structure might become amorphous when strain rate are higher than $10^{10} \mathrm{~s}^{-1}$ [33]. Similar kinds of results were obtained in [32]. 


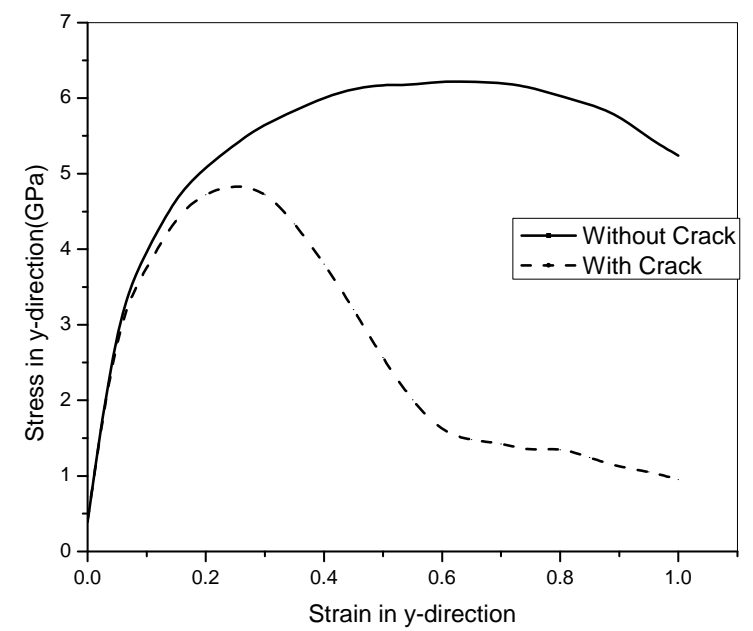

Fig.8. Influence of crack on Stress-Strain plot for Specimen 2

To identify the effect of crack on Cu-Zr specimen a separate simulation study has been done on specimen2, Table 1. It is clearly understood from the Fig.8 that the strength and toughness of CuZr alloy has drastically decreased by an initial edge crack. The peak stress for the without crack specimen is about 6.2 which decreased to 4.8 for the initial edge cracked specimen.

To circumvent the limited mechanical properties of the metallic glasses, the concept of developing a heterogeneous microstructure by combining a glassy matrix with crystalline second phase particles with a different length scale has recently been employed by several researchers. The aim of this approach is to restrict the rapid propagation of the shear bands by interaction with the ductile crystalline phases. This enables multiplication, branching, and restriction of the shear bands, thus controlling the instabilities otherwise responsible for early failure.

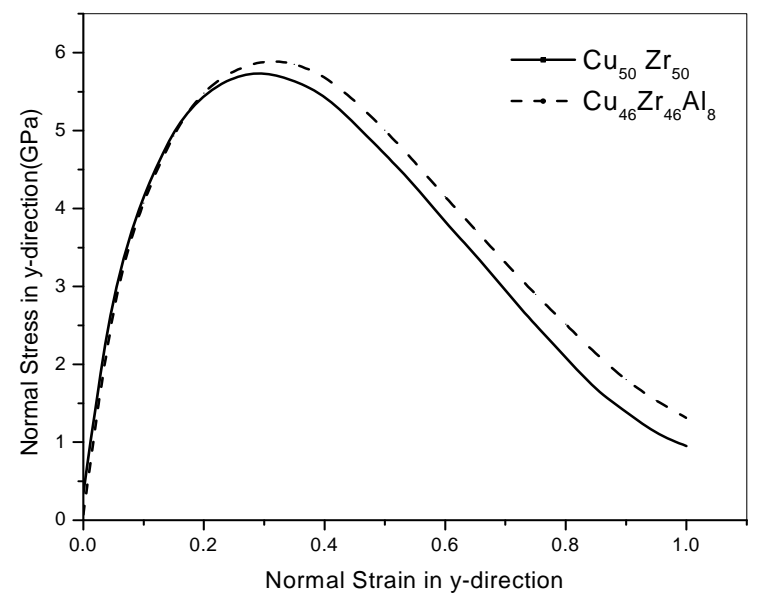

Fig.9. Stress-Strain plot for Cu-Zr BMG single crystalafter adding Al for specimen 2

In order to examine the above, Fig. 9 illustrates the stress-strain plot of $\mathrm{Cu}_{50} \mathrm{Zr}_{50}$ and $\mathrm{Cu}_{46} \mathrm{Zr}_{46} \mathrm{Al}_{8}$ BMGs. It is observed that the addition of $\mathrm{Al}$ in the $\mathrm{Cu}-\mathrm{Zr}$ alloy increases the peak stress, toughness as well as work hardenability. Similar kind of study has also been reported in the literature [28] where it has been observed that addition of $\mathrm{Al}$ into the alloy leads to an increased fracture strength.

\section{Crack Initiation and Propagation}

To further study the effect of $\mathrm{Al}$ on CuZr BMG, the simulated edge crack sample using MD simulation has been shown in graphic mode by using VMD. The sample has been loaded in mode-I tensile load. The snapshot of the crack growth simulation has been captured. Though the snapshot was taken at various time step, due to the limitation of space here only for a particular time step it has been illustrated. 


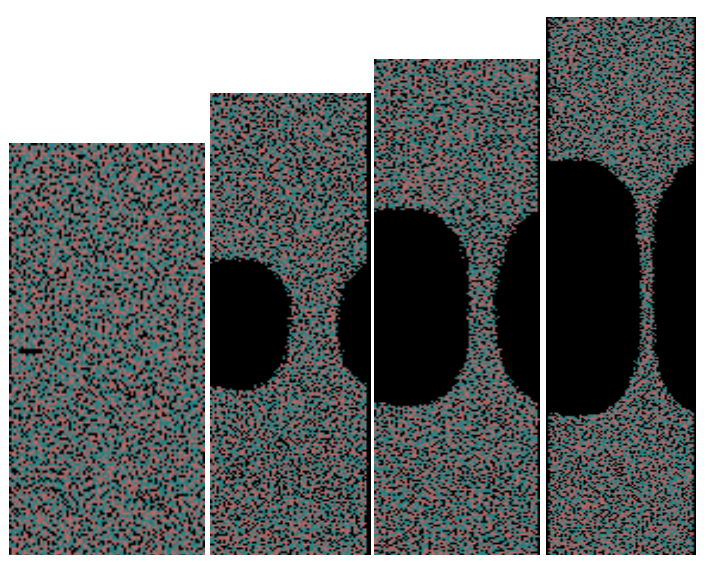

Fig.10. Crack growth of $\mathrm{Cu}_{50} \mathrm{Zr}_{50}$ Sample-2 at particular time steps

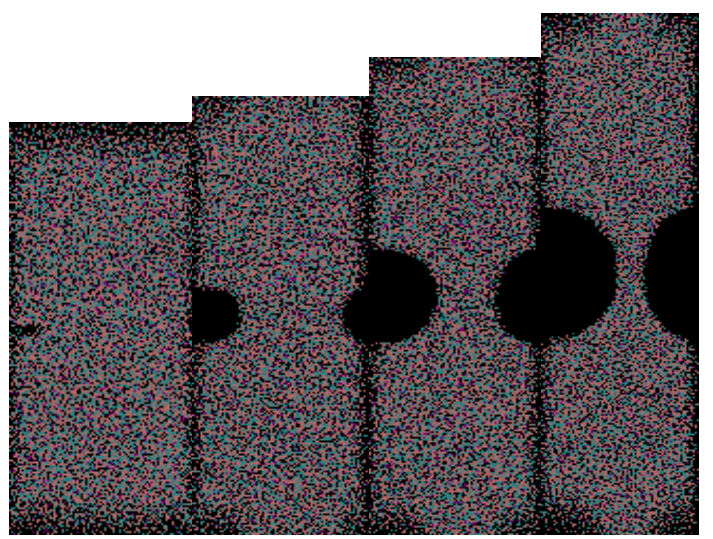

Fig.11. Crack growth of $\mathrm{Cu}_{46} \mathrm{Zr}_{46} \mathrm{Al}_{8}$ Sample-2 at particular time steps

In Fig.10 a sample of $\mathrm{Cu}_{50} \mathrm{Zr}_{50}$ of sample-2 size has been considered to study the crack propagation behaviour under tensile load. The crack starts growing just after the peak stress point is reached. The growth shows that there is no crack deviation and it propagates in mode-I configuration for the specimen. During the simulation it was also observed that crack propagated slowly in the start and rapidly in the end which finally lead to fracture.

From the Fig. 8 it was concluded that the addition of Al has a significance effect on the mechanical strength of $\mathrm{Cu}-\mathrm{Zr}$ alloy. So a comparative study has been done with $\mathrm{Cu}_{46} \mathrm{Zr}_{46} \mathrm{Al}_{8}$ alloy in Fig.11, which indicates that there is a percentage increase in the toughness after adding $\mathrm{Al}$, keeping other factors same. Some more detailed analysis should be done in future.

\section{CONCLUSION}

In the current study, the influence of specimen size, temperature, addition of $\mathrm{Al}$ and strain rate on the strength and fracture of monolithic Cu-Zr BMG was analysed via the MD simulations using EAM potential. The results clearly demonstrate that there is strong dependence of specimen size, temperature, addition of Al and strain rate on the averaged stress-strain responses. The peak stress point increases with increasing the above parameters though the initial slope of the average stress-strain curve is independent of the size, temperature and strain rate. The reasons for the above phenomena though explained at the appropriate places may be summarized in this way; increase in the peak stress value may be a) due to more free volume content and multiple shear bands, b) due to more blunting and crack arrest at high temperature, c) induction of second phase particles with a different length scale to restrict the rapid propagation of the shear bands and d) due to a greater degree of lattice disorder at higher strain rate. Because of limited computational resources, a full range comparison simulation data could not be provided. An integrated experimental, analytical and computational study is suggested in order to provide more useful information for further research towards this area. The simulation results presented here may be useful as guidelines for future research intended for better understanding the fracture behavior of the Cu-Zr BMG. 


\section{REFERENCES}

[1] Kitamura T, Hirakata H, Sumigawa T and Shimada T 2011, Fracture Nanomechanics (1st ed.) Pan Stanford Publishing, USA

[2] Tang T, Kim S, Horstemyer M F and Wang P 2011Engng. Fract. Mech.78 191-201.

[3] Paduraru A, Kenoufi A, Bailey N P and Schiotz J 2007 Adv. Engng. Mat.9 505-08.

[4] Abraham F E, Brodeck D, Rudge W E and XuX 1997J.Mech. Phys. Solids45 1595-1619

[5] Swadener J G, Baskes M I and Nastasi M 2002 Phys. Rev. Lett89 085503 (4 pages)

[6] Hauch J A, Holland D, Marder M P and Swinney H L 1999 Phys. Rev. Lett.82 3823-26

[7] Falk M L 1999 Phys. Rev. B60 7062-70

[8] Abraham F F, Scheneider D, Rafey R A, Rudge W E 1997 J. Mechphys solids45 1461

[9] Abraham F F 2005 J. Mech. Phys. Solids53 1071-78

[10] Guo Y, Wang C and Zhao D 2003 Mater. Sci. Engng. A349 29-35

[11] Abraham F and Broughton J Q 1998 Comput. Mater. Sci.10 1-9

[12] Latapie A and Farkas D 2003 Model. Simul.Mater. Sci. Engng.11 745-53

[13] Farkas D, Swygenhoven H V and Derlet P M 2002 Phys. Rev. B66 060101-1

[14] Luque A, Aldazabal J, Martinez-Esnaola J M and Sevillano J G 2007 Fatigue Fract. Engng.Mater.Struct.30 1008-15

[15] Zhou S J, Lomdahl P S, Voter A F and Holian B L 1998 Engng. Fract. Mech.61 173-87.-----

[16] Rafii-Tabar H, Shodja H M, Darabi, M and Dahi A 2006 Mech. Mater.38 243-52.

[17] Xu S W and Deng X M 2008 Nanotechnology19 115705.

[18] Sun D Y, Mendelev M I, Becker C A, Kudin K, Haxhimali T and Asta M 2006 Phys. Rev. B73 (2) 024116 (12 pages)

[19] Xu J, Ramamurty U and Ma E 2010 JOM 62 (4) 10-18.

[20] Hu Y, Yan H H, Lin T, Li J F and Zhou Y H 2012 J. Alloys. Compd.527 36-39

[21] Li C, Kou S, Zhao Y, Liu G and Ding Y 2012 Prog. Nat. Sc. Mat. Int.22(1) 21-25

[22] Ogata S, Shimizu F, Li J, Wakeda M and Shibutani Y 2006 Intermetallics 14 1033-37

[23] Schroers J and Johnson W L 2004 Phys. Rev. Lett.93 255506-1

[24] Das J, Tang M B, Kim K B, Theissmann R, Baier F, Wang W H and Eckert J 2005 Phys. Rev. Lett.94 205501-1

[25] Lammps 2011, http://lammps.sandia.gov

[26] Marian J and Knap J 2007 Int. J. Multiscale Comp. Engng.5 287-94

[27] Li C, Kou S, Zhao Y, Liu G, Ding Y 2012 Prog.Nat. Sc. Mat. Int.22(1) 21-25

[28] B.A. Sun, M.X. Pan, D.Q. Zhao, W.H. Wang, X.K. Xi, M.T. Sandor, Y. Wub 2008Acta Mater59 1159-1162

[29] Yong Gan and Chen J K 2009ApplPhys A95 357-62

[30] Warner D H and Curtin W A 2009 Acta Mater.57 4267-77.

[31] Ikeda H, Qi Y, Cagin T, Samwer C, Johnson W L and Goddard W A 1999 Phys. Rev. Lett.82 2900-03

[32] Koh S J A, Lee H P, Lu C and Cheng Q H 2005 Phys. Rev. B72 085414

[33] Sunny G, Lewandowski J and Prakash V 2007 J. Mater. Res.22(2) 389-401.

\section{AUTHOR PROFILE}

Mr. Rahul Chug is an Assistant Professor in Department of Mechanical \& Automation Engineering at Maharaja Agrasen Institute of Technology, New Delhi (India). He has completed his M.Tech. in Machine Design from IIT(BHU), Varanasi. He has a teaching experience of 03 Years.He has presented several papers in various Conferences.

Mr. Sachin Gupta is an Assistant Professor in Department of Mechanical \& Automation Engineering at Maharaja Agrasen Institute of Technology, New Delhi (India). He is also pursuing PhDfrom YMCA University of Science \& Technology, Faridabad, Haryana (India). He has completed his M.Tech. in Mechanical Engineering from NIT, Kurukshetra. He has a teaching experience of more than 10 Years. He has published several papers in reputed Journals and Conferences. 